\title{
Leserbrief
}

Peter Cerwenka

\section{Bestands- und Flussgrößen}

An dem außerordentlich begrüßenswerten Beitrag „Grundprobleme von Indikatorsystemen für Regionale Nachhaltigkeit" (RuR Heft 5-6/2001, S. 381-391) von Kilian Bizer und Rolf Sternberg, der durch sein ernsthaftes und redliches Bemühen um eine wissenschaftlich saubere Aufarbeitung des Problems inter- und intraregionaler Vergleichbarkeit von Nachhaltigkeit besticht und durch völlige Ideologiefreiheit aus dem Meer der interessengebundenen appellativen Papierflut zum Thema Nachhaltigkeit herausragt, möchte ich eine kleine Korrektur anbringen.

Ist es schon bemerkenswert, dass Autoren überhaupt zwischen Bestandsgrößen mit einer Absolutdimension zu einem Zeitpunkt t (z.B. $W_{\mathrm{t}}$ [ha Waldfläche]) und Flussgrößen mit einer zeitbezogenen Dimension (z.B. $\mathrm{w}(\mathrm{t})$ [ha/a jährliche Waldflächenveränderung]) unterscheiden, so ist aus meiner Sicht doch hinzuzufügen, daß es die dritte von Bizer/Sternberg genannte Gruppe „maßnahmenbezogene Größen” als zusätzliche trennscharfe Kategorie nicht geben kann. Dies folgt aus der definitorischen Verknüpfung von Bestands- und Flussgrößen über die Zeitachse $t$.*

Bezeichnet man mit dem Index „0" die Bestandsgröße W zum Zeitpunkt $t_{0}$ und mit dem Index „1" jene zum Zeitpunkt $t_{1}$, dann gilt definitionsgemäß:

$$
W_{1}=W_{0}+\int^{t_{1}} \mathrm{w}(t) \cdot d t
$$

$t_{0}$

Die Flussgröße w, also die zeitliche Veränderung w(t) der Bestandsgröße $W_{t}$ (in unserem Fall der Waldfläche), kann sich allerdings (gezeigt am Beispiel des Waldes) aus zwei Komponenten zusammensetzen, nämlich einer natürlichen $w_{a}$ und einer künstlichen (also „maßnahmenverursachten") $w_{b}$ :

$$
w(t)=w_{a}(t)+w_{b}(t)
$$

$w_{a}(t)$ : Differenz aus natürlichem Zuwachs und natürlichem Absterben des Waldes

$\mathrm{w}_{\mathrm{b}}(\mathrm{t})$ : Differenz aus (künstlicher) Aufforstung und (künstlicher) Abholzung des Waldes

Ist die Flussgröße $\mathrm{w}_{\mathrm{a}}(\mathrm{t})$ im Allgemeinen ein stetiger, nach natürlichen Gesetzmäßigkeiten ablaufender Prozess, so ist die Flussgröße $w_{b}(t)$ im Allgemeinen eine

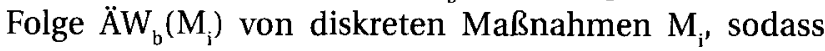
man (1) auch wie folgt anschreiben kann:

$$
\mathrm{W}_{1}=\mathrm{W}_{0}+\int_{\mathrm{t}_{0}}^{\mathrm{t}_{1}} \mathrm{~W}_{\mathrm{a}}(\mathrm{t}) \cdot \mathrm{dt}+\sum_{\mathrm{i}=\mathrm{l}}^{\mathrm{n}} \Delta \mathrm{W}_{\mathrm{b}}\left(\mathrm{M}_{\mathrm{i}}\right)
$$

$\mathbf{n}$ ist dabei die Anzahl der in das Zeitintervall $\left(t_{0}, t_{1}\right)$ fallenden Maßnahmen $\mathrm{M}_{\mathrm{i}}$.

Daraus erhellt, dass die von Bizer/Sternberg genannte eigenständige Kategorie „maßnahmenbezogene Größen" nicht neben Bestands- und Flussgrößen eine dritte Kategorie darstellt, sondern eine Teilkategorie der Flussgrößen. Ein Drittes gibt es nicht.

\section{Anmerkung}

* Siehe hierzu: Cerwenka, P.: Nachhaltiger Verkehr? In: Der Nahverkehr, 18 (2000), Nr. 5, S. 30-34

Prof. Dr. Peter Cerwenka Institut für Verkehrssystemplanung TU Wien Gusshausstraße 3/269 A 1040 Wien 